\title{
Eradication of chronic Helicobacter pylori infection by therapeutic vaccination
}

Ghiara P, Rossi M, Marchetti M, et al. Therapeutic intragastric vaccination against Helicobacter pylori in mice eradicates an otherwise chronic infection and confers protection against reinfection. Infect Immun 1997;65:4997-5002.

\section{Abstract}

Chronic infection of the gastroduodenal mucosae by the gram-negative spiral bacterium Helicobacter pylori is responsible for chronic active gastritis, peptic ulcers, and gastric cancers such as adenocarcinoma and low-grade B-cell lymphoma. The success of eradication by antibiotic therapy is being rapidly hampered by the increasing occurrence of antibiotic-resistant strains. An attractive alternative approach to combat this infection is represented by the therapeutic use of vaccines. In the present work, we have exploited the mouse model of persistent infection by mouse-adapted $H$.pylori strains that we have developed to assess the feasibility of the therapeutic use of vaccines against infection. We report that an otherwise chronic $H$. pylori infection in mice can be successfully eradicated by intragastric vaccination with $H$. pylori antigens such as recombinant $\operatorname{VacA}$ and $\operatorname{CagA\text {,which}}$ were administered together with a genetically detoxified mutant of the heat-labile exterotoxin of Escherichia coli (referred to as LTK63), in which the serine in position 63 was replaced by a lysine. Moreover, we show that therapeutic vaccination confers efficacious protection against reinfection. These results represent strong evidence of the feasibility of therapeutic use of VacA- or CagA-based vaccine formulations against $H$. pylori infection in an animal model and give substantial preclinical support to the application of this kind of approach in human clinical trials.

\section{Comment}

Effective antibiotic based therapies for eradicating Helicobacter pylori have been developed in recent years. ${ }^{12}$ There is, however, an the increasing problem of antibiotic resistance in $\mathrm{H}_{\text {pylori }}{ }^{3}$ and in the long term the consequence of large scale eradication programmes could be a reduction in the efficacy of current antibiotic based regimens.

The development of a vaccine against $H$ pylori which confers long term protective immunity is the best strategy to circumvent the problem of antibiotic resistance and to eradicate $H$ pylori on a global scale. The feasibility of inducing protective immune responses to helicobacter by oral vaccination with bacterial antigens and a mucosal adjuvant was initially demonstrated in the $H$ felis murine model. ${ }^{4-6}$ Vaccination with both $H$ pylori urease ${ }^{5}$ and heat shock proteins (HspA and $\mathrm{HspB})^{6}$ protected against subsequent challenge with $H$ felis. However, $H$ felis lacks many of the virulence factors present in $H$ pylori, such as the cag pathogenicity island ${ }^{7}$ and the cytotoxin VacA, ${ }^{8}$ precluding analysis of these antigens as candidate vaccines in the $H$ felis model. The development of mouse adapted $H$ pylori strains which cause chronic infection in mice was a major advance. ${ }^{9}$ The $H$ pylori mouse model has permitted the testing of vaccines containing purified $H$ pylori antigens against homologous challenge infection. ${ }^{9}$ To date, a number of protective $H$ pylori antigens have been identified which confer immunity against $H$ pylori infection in the mouse, including purified VacA, ${ }^{9}$ urease, ${ }^{9} \mathrm{CagA},{ }^{10}$ and catalase. ${ }^{11}$

The prophylactic vaccination studies in animals showed that, in contrast to natural infection, protective immune responses to gastric helicobacter can be induced. The question was then addressed whether vaccination could be used to eliminate existing infection. Oral immunisation with helicobacter sonicates ${ }^{12}$ or recombinant urease B subunit ${ }^{13}$ together with cholera toxin eliminated chronic $H$ felis infection in mice and protected against subsequent $H$ felis challenge. ${ }^{13}$ Therapeutic immunisation also was successful in ferrets infected with $\mathrm{H}$ mustelae. ${ }^{14}$

In their study Ghiara et al investigated the feasibility of the therapeutic use of $H$ pylori antigens as vaccines against chronic $H$ pylori infection. Importantly, they also test the ability of a genetically detoxified mutant of the heat labile toxin of Escherichia coli (LTK63) ${ }^{15}$ to act as a mucosal adjuvant. Their study shows for the first time that oral administration of either $H$ pylori sonicates or recombinant proteins (VacA and CagA), together with LTK63, successfully eradicates $H$ pylori infection in mice. The treated mice remained non-infected for at least three months after therapeutic vaccination, confirming long term persistence of eradication rather than suppression of the chronic infection. Importantly, Ghiara et al also show that the therapeutic vaccine both eradicates infection and confers protection against subsequent challenge.

Successful mucosal vaccination requires strong adjuvants to improve the poor immunogenicity of coadministered antigens. A key role of mucosal adjuvants is likely to be the stimulation of $\mathrm{T}$ helper-2 (Th2) type mucosal responses. ${ }^{16}{ }^{17}$ The inherent toxicity of the mucosal adjuvants cholera toxin and heat labile enterotoxin (LT) has been a major limitation for their use as vaccines in humans. Recent clinical studies in $H$ pylori infected human volunteers testing the safety and immunogenicity of recombinant $H$ pylori urease showed that the coadministration of LT was associated with a high incidence of diarrhoea. ${ }^{18}$ The use of genetically detoxified heat labile enterotoxins such as LTK $63^{15}$ is likely to circumvent this problem. LTK63 has a single amino acid substitution (Ser to Lys in position 63) which destroys its ADP ribosylating toxic activity. ${ }^{15}$ Non-toxic LTK63 has been used success- 
fully as a mucosal adjuvant in animal models to induce antigen specific humoral responses ${ }^{19}$ and measles virus specific cytotoxic lymphocyte responses. ${ }^{20}$ The demonstration by Ghiara et al that the genetically detoxified mutant of the heat labile $E$ coli enterotoxin is also suitable for therapeutic oral vaccination against $H$ pylori is an important development for its future clinical use.

An understanding of the mechanisms involved in the induction of protective mucosal responses to $H$ pylori is important for future clinical use of prophylactic and therapeutic vaccines. As discussed by Ghiara et al, the role of the adjuvant in therapeutic vaccination may be to change the nature of the chronic gastric Th1 type tissue damaging response to a $\mathrm{Th} 0$ or $\mathrm{Th} 2$ protective response. In the $\mathrm{H}$ felis mouse model stimulation of Th2 responses has been associated with a reduction in both bacterial load ${ }^{21}$ and gastric inflammation. ${ }^{22}$ Down-regulation of Th1 responses by neutralisation of interferon- $\gamma$ in $H$ felis immunised mice resulted in unmasking of both splenic and gastric interleukin 4 (IL-4) Th2 responses. ${ }^{22}$ Adoptive transfer of splenic $T$ cells from mice after immunisation and challenge and of in vitro generated $H$ felis specific Th2 cell lines also reduced the bacterial load of $H$ felis after challenge in naive recipients. ${ }^{21}$ Consistent with these observations, Mohammadi et al also found that IL-4 knockout mice had an increased bacterial load of $H$ felis compared with wild type controls. ${ }^{21}$

Ghiara et al, while confirming that chronic $H$ pylori infection in the mouse model induces a Th1 response, did not examine the effector mechanisms contributing to the success of therapeutic vaccination. They speculate, however, that therapeutic vaccination induces activation of Th0 or Th2 responses which in turn trigger bacterial eradication. Current evidence suggests that gastric Th1 responses predominate in humans with chronic $H$ pylori infection. ${ }^{2324}$ It remains to be investigated whether the human gastrointestinal responses can be similarly modified. If so, given the availability of non-toxic mucosal adjuvants, ${ }^{15}$ therapeutic vaccines may prove to be a novel means of eradicating $H$ pylori and a therapeutic alternative to the use of antibiotic based regimens.

Molecular Medicine Unit, Level 7,

J E CRABTREE

Clinical Sciences Building,

St fames's University Hospital,

Leeds LS9 7TF, UK

1 Bazzoli F, Zagari RM, Fossi S, et al. Short-term low-dose triple therapy for the eradication of Helicobacter pylori. Eur 7 Gastroenterol Hepatol 1994;6:773-777.
2 Bazzoli F, Zagari M, Pozzato P, et al. Evaluation of short-term low-dose triple therapy for the eradication of $\mathrm{H}$. pylori by factorial design in a randomized, double blind, controlled study. Aliment Pharmacol Ther 1998 (in press)

3 Megraud F. Resistance of Helicobacter pylori to antibiotics. Aliment Pharmacol Ther 1997;11(suppl 1):43-53.

4 Chen M, Lee A, Hazell S. Immunisation against gastric Helicobacter infection in a mouse Helicobacter felis model. Lancet 1992;339:1120-1.

5 Michetti P, Corthesy-Theulaz I, Davin C, et al. Immunization of BALB/c mice against Helicobacter felis infection with $\mathrm{H}$. pylori urease. Gastroenterology 1994;107:1002-11.

6 Ferraro RL, Thiberge JM, Kansau I, et al. The GroES homolog of Helicobacter pylori confers protective immunity against mucosal infection in mice. Proc Natl Acad Sci USA 1995;92:6499-503.

7 Censini S, Lange C, Xiang ZY, et al. cag, a pathogenicity island of Helicobacter pylori, encodes type I-specific and disease-associated virulence factors. Proc Natl Acad Sci USA 1996;93:14648-53.

8 Telford JL, Ghiara P, Dell'Orco M, et al. Gene structure of Helicobacter pylori cytotoxin and evidence of its key role in gastric disease. $\mathcal{J}$ Exp Med 1994;179:1653-8.

9 Marchetti M, Arico B, Burroni D, et al. Development of a mouse model of Helicobacter pylori infection which mimics human disease. Science 1995;267:1655-8.

10 Marchetti M, Rossi M, Giannelli V, et al. Protection against Helicobacter pylori infection in mice by intragastric vaccination with $\mathrm{H}$. pylori antigens is achieved using a non-toxic mutant of E. coli heat-labile enterotoxin (LT) as adjuvant. Vaccine 1998;16:33-7.

11 Radcliff FJ, Hazell S, Kolesnikow T, et al. Catalase, a novel antigen for Helicobacter pylori vaccination. Infect Immun 1997;65:4668-74.

12 Doidge C, Gust I, Lee A, et al. Therapeutic immunisation against Helicobacter infection. Lancet 1994;343:914-15.

13 Corthésy-Theulaz I, Porta N, Glauser M, et al. Oral immunization with Helicobacter pylori urease B subunit as a treatment against Helicobacter infection in mice. Gastroenterology 1995;109:115-21.

14 Cuenca R, Blanchard TG, Czinn SJ, et al. Therapeutic immunization against Helicobacter mustelae in naturally infected ferrets. Gastroenterology 1996:110:1770-5.

15 Pizza M, Fontana MR, Giuliana MM, et al. A genetically detoxified derivative of heat-labile Escherichia coli enterotoxin induces neutralizing antibodies against the A subunit. $\mathcal{F} \operatorname{Exp}$ Med 1994;180:2147-53.

16 Yamamoto S, Kiyono H, Yamamoto M, et al. A nontoxic mutant of cholera toxin elicits Th2-type responses for enhanced mucosal immunity. Proc Natl Acad Sci USA 1997;94:5267-72.

17 Marinaro M, Staats HF, Hiroi T, et al. Mucosal adjuvant effect of cholera toxin in mice results from induction of T helper 2 (Th2) cells and IL-4. $\mathcal{F}$ Immunol 1995;155:4621-9.

18 Michetti $\mathrm{P}$, Kreiss C, Kotloff $\mathrm{K}$, et al. Oral immunization of $\mathrm{H}$. pylori infected adults with recombinant urease and LT adjuvant [abstract]. Gastroenterology 1997;112:A1042.

19 Di Tomamaso A, Saletti G, Pizza M, et al. Induction of antigen-specific antibodies in vaginal secretions by using nontoxic mutant of heat-labile enterotoxin as a mucosal adjuvant. Infect Immun 1996;64:974-9.

20 Partidos CD, Pizza M, Rappuoli R, et al. The adjuvant effect of a non-toxic mutant of heat-labile enterotoxin of Escherichia coli for the induction of virus-specific CTL responses after intranasal co-immunization with a synthetic peptide. Immunology 1996;89:483-7.

21 Mohammadi M, Nedrud J, Redline R, et al. Murine CD4 T-cell response to Helicobacter infection: TH1 cells enhance gastritis and TH2 cells reduce bacterial load. Gastroenterology 1997;113:1848-57.

22 Mohammadi M, Czinn S, Redline R, et al. Helicobacter-specific cell-mediated immune responses display a predominant Th1 phenotype and promote a delayed-type hypersensitivity response in the stomachs of and promote a delayed-type hypers

23 Karttunen R, Kartunnen T, Ekre HP, et al. Interferon gamma and interleukin-4 secreting cells in the gastric antrum in Helicobacter pylori positive and negative gastritis. Gut 1995;36:341-5.

24 D'Elios MM, Manghetti M, De Carli M, et al. T helper 1 effector cells specific for Helicobacter pylori in the gastric antrum of patients with peptic ulcer disease. F Immunol 1997;158:962-7. 